\title{
Web matrices: structural properties and generating combinatorial identities
}

\author{
Mark Dukes \\ Computer \& Information Sciences \\ University of Strathclyde \\ Glasgow, G1 1XH, UK. \\ mark.dukes@strath.ac.uk
}

\author{
Chris D. White \\ School of Physics and Astronomy \\ University of Glasgow \\ Glasgow, G12 8QQ, UK \\ c.white@physics.gla.ac.uk
}

Submitted: Oct 13, 2015; Accepted: Feb 14, 2016; Published: Mar 4, 2016

Mathematics Subject Classifications: 05A05, 05A19, 05C50

\begin{abstract}
In this paper we present new results for the combinatorics of web diagrams and web worlds. These are discrete objects that arise in the physics of calculating scattering amplitudes in non-abelian gauge theories. Web-colouring and web-mixing matrices (collectively known as web matrices) are indexed by ordered pairs of webdiagrams and contain information relating the number of colourings of the first web diagram that will produce the second diagram.

We introduce the black diamond product on power series and show how it determines the web-colouring matrix of disjoint web worlds. Furthermore, we show that combining known physical results with the black diamond product gives a new technique for generating combinatorial identities. Due to the complicated action of the product on power series, the resulting identities appear highly non-trivial.

We present two results to explain repeated entries that appear in the web matrices. The first of these shows how diagonal web matrix entries will be the same if the comparability graphs of their associated decomposition posets are the same. The second result concerns general repeated entries in conjunction with a flipping operation on web diagrams.

We present a combinatorial proof of idempotency of the web-mixing matrices, previously established using physical arguments only. We also show how the entries of the square of the web-colouring matrix can be achieved by a linear transformation that maps the standard basis for formal power series in one variable to a sequence of polynomials. We look at one parameterized web world that is related to indecomposable permutations and show how determining the web-colouring matrix entries in this case is equivalent to a combinatorics on words problem.
\end{abstract}

Keywords: web diagram, web world, combinatorial identity, idempotence, black diamond product 


\section{Introduction}

Web diagrams are discrete objects that are subject to certain colouring and reconstruction operations, and arise in physics in the calculation of scattering amplitudes in non-abelian gauge theories $[9,16,20]$. A prominent example is the theory of quarks and gluons, Quantum Chromodynamics (QCD), which is of great topical relevance given its application to current experiments such as the Large Hadron Collider.

Whilst some properties of web-mixing matrices have been established from a physics point of view $[9,12]$, a fully general understanding of their structure and properties remains elusive. Web-colouring and web-mixing matrices admit a purely combinatorial definition and this provides an alternative framework to the physical picture to elucidate their properties. Our ultimate hope is that a detailed understanding of web diagrams and their matrices can be used to dramatically improve the precision of theoretical predictions for particle collider experiments.

The combinatorics of web diagrams and web matrices is an interesting study in its own right in that it combines parts of order theory, graph theory, and the theory of permutations in a new and novel way. Our seminal paper on the combinatorics of web diagrams [5] looked at these objects from several angles and has a companion physics paper [4] which explains how the results are pertinent to particle physics applications ${ }^{1}$. We were able to show, for example, that for particular web diagrams, the diagonal entries of the web matrices corresponding to them depend on the generating function of the descent statistic formed by summing over the Jordan-Hölder set of all linear extensions of a web diagram's decomposition poset (partially ordered set). We examined some special (parameterized) classes of web worlds which could be 'exactly' solved, and one of these saw the introduction of a new permutation statistic that did not seem to have been previously studied. Another result is that the number of different diagrams in a web world can be given by a hook-length style formula on its representation matrix.

Our aim in this study is not just to build on some of the results and directions that were initiated in [5], but to investigate some new aspects of these web diagrams that have not been explored before. Amongst these, we will give a combinatorial proof of idempotency of the web-mixing matrices that was originally established by way of a physics argument in Gardi and White $[12, \S 3]$. We will also introduce the black diamond product on power series and show how the web-colouring matrix of a web world whose web diagrams can be 'partitioned' (in a sense to be made precise later) depend on this black diamond product. Furthermore, we will show how a physics argument gives rise to a new technique for generating combinatorial identities using the black diamond product. We think that this technique is interesting in that it has the potential to generate quite unusual looking identities due to the black diamond product behaving in quite a complicated way on the power series in question.

We will concern ourself with the web diagrams that were defined in $[5]^{2}$. Let $P=$

\footnotetext{
${ }^{1}$ For a recent introduction to the latter, see ref. [22].

${ }^{2}$ The diagrams we consider here are referred to as Multiple Gluon Exchange Webs (MGEWs) in refs. $[8,10]$. Other types of web are possible, but are beyond the scope of the present work.
} 
$\left\{p_{1}, \ldots, p_{n}\right\}$ be a set of $n$ pegs that are rooted to a plane. A web diagram $D$ on $P$ is a set of 4 -tuples $e_{i}=\left(a_{i}, b_{i}, c_{i}, d_{i}\right)$ where the $e_{i}$ represents an edge between peg $p_{a_{i}}$ and peg $p_{b_{i}}$ for which the endpoint of $e_{i}$ on peg $p_{a_{i}}$ is the $c_{i}$ th highest vertex on that peg from the plane, and the endpoint of $e_{i}$ on peg $b_{i}$ is the $d_{i}$ th highest vertex on that peg from the plane. The labels of the endpoints of edges on each peg must be distinct, and this is taken care of in the formal definition, Definition 1. We will sometimes abuse the terminology by referring to 'peg $i$ ' in place of 'peg $p_{i}$ '.

Two illustrations of web diagrams are given in Figure 1. The web world of a web diagram $D$ is the set of all web diagrams that result from permuting the order in which vertices appear on a peg, and we usually denote it by $W(D)$. The two web diagrams in Figure 1 are in the same web world. In the physics context in which such diagrams arise, the pegs represent quarks or gluons emanating from the interaction point, and the lines joining pegs correspond to additional gluons being radiated and absorbed. The conventional depiction of each web diagram is a so-called Feynman diagram, an example of which is shown on the left of Figure 2.

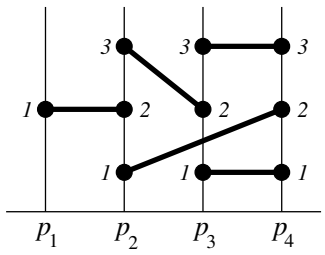

(a)

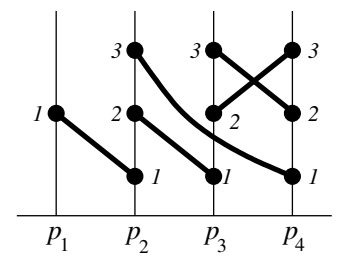

(b)

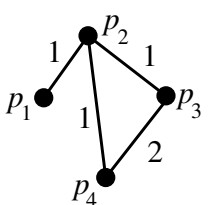

(c)

Figure 1: Two examples of web diagrams in the same web world. Web diagrams (a) and (b) in this figure both have a single edge between 'pegs' $p_{2}$ and $p_{4}$. However the heights of the endpoints differ in both diagrams. In (a) the heights of this edge on pegs $p_{2}$ and $p_{4}$ are, respectively, 1 and 2 . We represent this edge by the 4 -tuple $(2,4,1,2)$. In (b) the heights of this edge are, respectively, 3 and 1 . The edge in this diagram is represented by the 4 -tuple $(2,4,3,1)$. The web graph of the web world (to be defined in Section 2) containing the web diagrams is shown as (c).

To every web world $W$ we associate two important matrices, $\mathfrak{M}^{(W)}$ and $\mathfrak{R}^{(W)}$, called the web-colouring and web-mixing matrices, respectively. The entries of these matrices are indexed by ordered pairs of web diagrams, and contain information about colourings of the edges of the first web diagram that yield the second diagram under a construction determined by a colouring.

In this paper we deepen our study of these recently defined structures, with the goal of ascertaining general properties. Section 2 contains some background and definitions necessary for the rest of the paper. In Section 3 we introduce the black diamond product on formal power series and prove a decomposition theorem for the web matrices of web worlds that may be partitioned into web worlds on disjoint peg sets. In Section 4 we give a new method for generating combinatorial identities by exploiting some physical properties of web worlds. 
In Section 5 we give two new results to explain the repeated entries that one notices occurring in web matrices. The first of theses results concerns diagonal entries and shows that if the comparability graphs of the decomposition posets of two web diagrams (that satisfy a further technical condition) are the same, then the diagonal entries on the web matrices for these two diagrams will be the same. The second result explains how the action of flipping a web diagram upside-down combines with the colouring and reconstruction operations on that same web diagram. In Section 6 we derive an expression for the entries of the square of a web-colouring matrix in terms of the entries of the web-colouring matrix, and give a combinatorial proof that the web-mixing matrices are idempotent.

Finally, in Section 7 we investigate a particular web world whose diagrams consist of only two pegs that have multiple edges between them. We show how they are related to indecomposable permutations and how calculating the entries of the web matrices for this web world reduces to a combinatorics on words problem.

\section{Definitions and terminology}

Let $\mathbb{C}[[x]]$ be the ring of formal power series in the variable $x$ over the ring $\mathbb{C}$. Given $f \in \mathbb{C}[[x]]$ we will denote by $\left[x^{i}\right] f$ the coefficient of $x^{i}$ in $f$ and we extend this notation to the multivariate case. For integers $a, b \in \mathbb{N}$ let $a \vee b$ denote the maximum of $a$ and $b$. If $a \leqslant b$, then let $[a, b]=\{a, a+1, \ldots, b\}$.

Although web diagrams were defined in Section 1, we give here the formal specification in order to remove any uncertainty surrounding their definition.

Definition 1. A web diagram on $n$ pegs having $m$ edges is a collection $D=\left\{e_{j}=\right.$ $\left.\left(a_{j}, b_{j}, c_{j}, d_{j}\right): 1 \leqslant j \leqslant m\right\}$ of 4 -tuples that satisfy the following properties:

(i) $1 \leqslant a_{j}<b_{j} \leqslant n$ for all $j \in[1, m]$.

(ii) For $i \in[1, n]$ let $p_{i}(D)$ be the number of $j$ such that $a_{j}$ or $b_{j}$ equals $i$, that is, the number of edges in $D$ incident with peg $i$. Then the labels of the vertices on peg $i$ must be the first $p_{i}(D)$ positive integers, i.e.

$$
\left\{d_{j}: b_{j}=i\right\} \cup\left\{c_{j}: a_{j}=i\right\}=\left[1, p_{i}(D)\right],
$$

and no vertex on a peg can be both a left endpoint and a right endpoint of an edge, i.e.

$$
\left\{d_{j}: b_{j}=i\right\} \cap\left\{c_{j}: a_{j}=i\right\}=\varnothing .
$$

We write $\operatorname{Pegs}(D)=\left(p_{1}(D), \ldots, p_{n}(D)\right)$. The labels of the $p_{i}(D)$ vertices on peg $i$ when read from bottom to top are $\left(1, \ldots, p_{i}(D)\right)$.

Given a web diagram $D=\left\{e_{j}=\left(a_{j}, b_{j}, c_{j}, d_{j}\right): 1 \leqslant j \leqslant m\right\}$, the set of pegs that are incident with at least one edge is $\operatorname{PegSet}(D)=\left\{a_{1}, \ldots, a_{m}, b_{1}, \ldots, b_{m}\right\}$.

A web diagram $D$ may be mapped to a simple graph $G([1, n], E)$ where $E(G)=$ $\{\{a, b\}:(a, b, c, d) \in D\}$. Web worlds are in 1-1 correspondence with loop-free simple graphs whose edges have labels in the set of positive integers. We call the labeled graph 

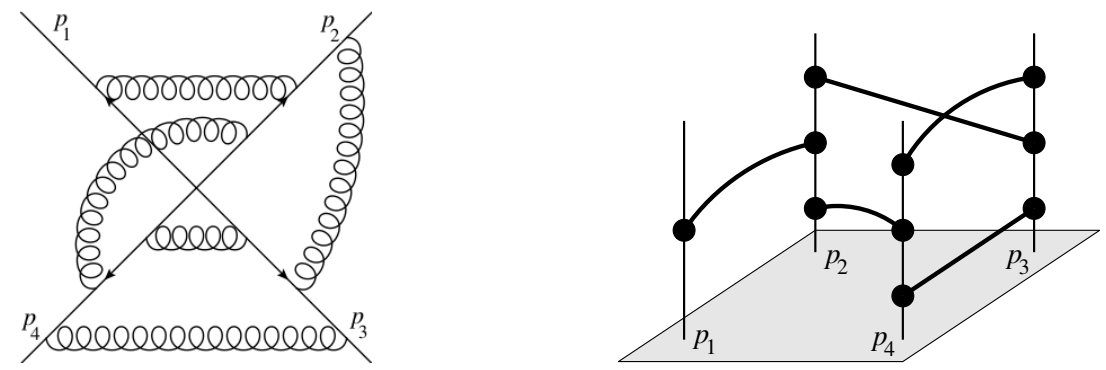

Figure 2: The Feynman diagram corresponding to the web diagram of Figure 1(a) is illustrated on the left. The gluon lines correspond to the edges of the web diagram. The heights of the endpoints correspond to their distance from the meeting points of the the 4 arrowed lines. The 'pegs and plane' representation is shown to its right.

associated with a web world its web graph. The vertices of the web graph correspond to the pegs of the web diagram (all vertices on a peg in the web diagram are projected to a single vertex in the web graph). The edges of the web graph are labeled with the multiplicities of edges between the pegs in the web diagram. The web world that the web diagrams (a) and (b) of Figure 1 are members of is represented by the web graph (c).

Definition 2. Let $D=\left\{e_{j}=\left(x_{j}, y_{j}, a_{j}, b_{j}\right): 1 \leqslant j \leqslant m\right\}$ and $D^{\prime}=\left\{e_{j}^{\prime}=\left(x_{j}^{\prime}, y_{j}^{\prime}, a_{j}^{\prime}, b_{j}^{\prime}\right)\right.$ : $\left.1 \leqslant j \leqslant m^{\prime}\right\}$ be two web diagrams with PegSet $(D), \operatorname{PegSet}\left(D^{\prime}\right) \subseteq\{1, \ldots, n\}$. The sum $D \oplus D^{\prime}$ is the web diagram obtained by placing the diagram $D^{\prime}$ on top of $D$;

$$
D \oplus D^{\prime}=D \cup\left\{\left(x_{j}^{\prime}, y_{j}^{\prime}, a_{j}^{\prime}+p_{x_{j}^{\prime}}(D), b_{j}^{\prime}+p_{y_{j}^{\prime}}(D)\right): 1 \leqslant j \leqslant m^{\prime}\right\} .
$$

If there exist two non-empty web diagrams $E$ and $F$ such that $D=E \oplus F$ then we say that $D$ is decomposable. Otherwise we say that $D$ is indecomposable.

Example 3. Consider the following two web diagrams: $D_{1}=\{(1,4,1,1),(2,6,1,2)$, $(2,6,2,1)\}$ and $D_{2}=\{(1,2,1,1),(3,5,1,1),(5,6,2,1)\}$. For $D_{1}$ we have

$$
\left(p_{1}\left(D_{1}\right), \ldots, p_{6}\left(D_{1}\right)\right)=(1,2,0,1,0,2)
$$

and so

$$
\begin{aligned}
& D_{1} \oplus D_{2} \\
&=\{(1,4,1,1),(2,6,1,2),(2,6,2,1)\} \cup\left\{\left(1,2,1+p_{1}\left(D_{1}\right), 1+p_{2}\left(D_{1}\right)\right),\right. \\
&\left.\left(3,5,1+p_{3}\left(D_{1}\right), 1+p_{5}\left(D_{1}\right)\right),\left(5,6,2+p_{5}\left(D_{1}\right), 1+p_{6}\left(D_{1}\right)\right)\right\} \\
&=(1,4,1,1),(2,6,1,2),(2,6,2,1)\} \cup\{(1,2,1+1,1+2),(3,5,1+0,1+0), \\
&(5,6,2+0,1+2)\} \\
&=\{(1,4,1,1),(2,6,1,2),(2,6,2,1),(1,2,2,3),(3,5,1,1),(5,6,2,3)\} .
\end{aligned}
$$

A $k$-colouring $\alpha$ of a web diagram $D$ is an assignment of the numbers $\{1, \ldots, k\}$ to the edges of $D$ such that the mapping $\alpha: D \mapsto\{1, \ldots, k\}$ is surjective. In this instance we 

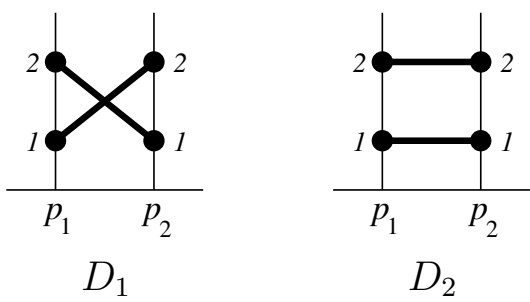

Figure 3: The two web diagrams of the web world in Example 4.

write $|\alpha|=k$. Let Colours $_{k}(W)$ be the set of $k$-colourings of web diagrams in $W=W(D)$, and $\operatorname{Colours}(W)=\operatorname{Colours}_{1}(W) \cup$ Colours $_{2}(W) \cup \cdots$.

The construction procedure that we alluded to in the Introduction happens in the following way: a $\ell$-colouring $\alpha$ of a web diagram $D$ produces $\ell$ new sub-web diagrams $D_{\alpha}(1), \ldots, D_{\alpha}(\ell)$, each of which consists of only the edges having that designated colour. These $\ell$ diagrams are not yet web diagrams, but we can relabel the heights of the vertices on each of the pegs to make them into web diagrams. Let rel be this relabelling operation. The new web diagram $D^{\prime}=\operatorname{Recon}(D, \alpha)=\operatorname{rel}\left(D_{\alpha}(1)\right) \oplus \cdots \oplus \operatorname{rel}\left(D_{\alpha}(\ell)\right)$ is formed by stacking the diagrams on top of one another in increasing order of the colours.

The $\left(D_{1}, D_{2}\right)$ entries of the web-colouring and web-mixing matrices of $W$ are:

$$
\mathfrak{M}_{D_{1}, D_{2}}^{(W)}(x)=\sum_{\ell \geqslant 1} x^{\ell} f\left(D_{1}, D_{2}, \ell\right) \quad \text { and } \quad \mathfrak{R}_{D_{1}, D_{2}}^{(W)}=\sum_{\ell \geqslant 1} \frac{(-1)^{\ell-1}}{\ell} f\left(D_{1}, D_{2}, \ell\right),
$$

where $f\left(D_{1}, D_{2}, \ell\right)$ is the number of $\ell$-colourings $\alpha$ of $D_{1}$ which give $\operatorname{Recon}\left(D_{1}, \alpha\right)=D_{2}$. As stated in the introduction, these web-mixing matrices occur in the calculation of scattering amplitudes in QCD. More specifically, they occur in the exponents of amplitudes, and dictate how the colour charge and kinematic degrees of freedom of highly energetic quarks and gluons are entangled by the radiation of additional lower energy gluons.

Example 4. Let $D_{1}=\left\{e_{1}=(1,2,1,2), e_{2}=(1,2,2,1)\right\}$. The web world generated by $D_{1}$ is $W\left(D_{1}\right)=\left\{D_{1}, D_{2}\right\}$ where $D_{2}=\left\{e_{1}^{\prime}=(1,2,1,1), e_{2}^{\prime}=(1,2,2,2)\right\}$. These are illustrated in Figure 3. There are three different colourings of $D_{1}$ :

$$
\begin{array}{llll}
\alpha\left(e_{1}\right)=1 \quad \alpha\left(e_{2}\right)=1 & \Rightarrow & \operatorname{Recon}\left(D_{1}, \alpha\right)=D_{1} \\
\alpha\left(e_{1}\right)=1 \quad \alpha\left(e_{2}\right)=2 \quad \Rightarrow & \operatorname{Recon}\left(D_{1}, \alpha\right)=D_{2} \\
\alpha\left(e_{1}\right)=2 \quad \alpha\left(e_{2}\right)=1 \quad \Rightarrow & \operatorname{Recon}\left(D_{1}, \alpha\right)=D_{2}
\end{array}
$$

Consequently $\mathfrak{M}_{D_{1}, D_{1}}^{(W)}(x)=x^{1}$ and $\mathfrak{M}_{D_{1}, D_{2}}^{(W)}(x)=2 x^{2}$. Likewise there are three different colourings of $D_{2}$ :

$$
\begin{array}{llll}
\alpha\left(e_{1}^{\prime}\right)=1 \quad \alpha\left(e_{2}^{\prime}\right)=1 \quad & \Rightarrow & \operatorname{Recon}\left(D_{2}, \alpha\right)=D_{2} \\
\alpha\left(e_{1}^{\prime}\right)=1 \quad \alpha\left(e_{2}^{\prime}\right)=2 \quad \Rightarrow & \operatorname{Recon}\left(D_{2}, \alpha\right)=D_{2} \\
\alpha\left(e_{1}^{\prime}\right)=2 \quad \alpha\left(e_{2}^{\prime}\right)=1 \quad \Rightarrow & \operatorname{Recon}\left(D_{2}, \alpha\right)=D_{2}
\end{array}
$$

Consequently $\mathfrak{M}_{D_{2}, D_{1}}^{(W)}(x)=0$ and $\mathfrak{M}_{D_{2}, D_{2}}^{(W)}(x)=x^{1}+2 x^{2}$. Therefore 
$D$

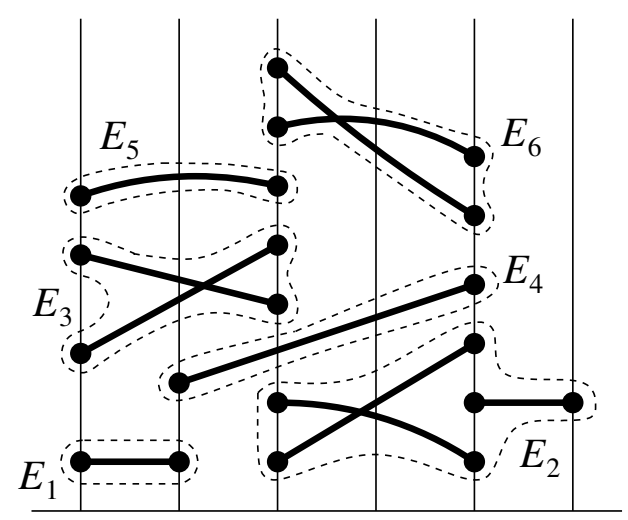

$P(D)$

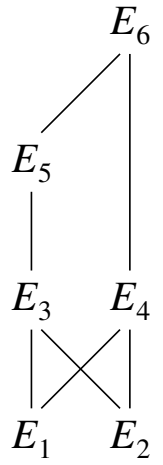

Figure 4: The decomposition poset of the web diagram in Example 6.

$$
\mathfrak{M}^{(W)}(x)=\left(\begin{array}{cc}
x & 2 x^{2} \\
0 & x+2 x^{2}
\end{array}\right) \quad \text { and } \quad \mathfrak{R}^{(W)}=\left(\begin{array}{cc}
1 & -1 \\
0 & 0
\end{array}\right) .
$$

The physical relevance of this example is that, owing to the second row of this matrix having no non-zero entries, only diagram $D_{1}$ survives in the exponents of scattering amplitudes. Further examples of these definitions and details can be found in $[5, \S 2]$.

Every web diagram $D$ may be decomposed and written as a sum of indecomposable web diagrams. A poset (partially ordered set) $P$ on the set of these constituent indecomposable web diagrams can be defined as follows:

Definition 5. Let $W$ be a web world and $D \in W$. Suppose that $D=E_{1} \oplus E_{2} \oplus \cdots \oplus E_{k}$ where each $E_{i}$ is an indecomposable web diagram. Let $P=\left\{E_{1}, \ldots, E_{k}\right\}$. Define the relation $<_{1}$ on $P \times P$ as follows: $E_{i}<_{1} E_{j}$ if $i<j$ and $\operatorname{PegSet}\left(E_{i}\right) \cap \operatorname{PegSet}\left(E_{j}\right) \neq \varnothing$. Let $<_{2}$ be the transitive closure of $<_{1}$ on $P \times P$ and let $\preceq$ be the reflexive closure of $<_{2}$ on $P \times P$. We call $P(D)=(P, \preceq)$ the decomposition poset of $D$.

Example 6. Consider the web diagram $D$ given on the left in Figure 4 . The poset $P(D)$ we get from this diagram illustrated to its right. The constituent indecomposable web diagrams are:

$$
\begin{aligned}
& E_{1}=\{(1,2,1,1)\} \\
& E_{2}=\{(3,5,1,3),(3,5,2,1),(5,6,2,1)\} \\
& E_{3}=\{(1,3,1,2),(1,3,2,1)\} \\
& E_{4}=\{(2,5,1,1)\} \\
& E_{5}=\{(1,3,1,1)\} \\
& E_{6}=\{(3,5,1,2),(3,5,2,1)\} .
\end{aligned}
$$

In the following sections we will repeatedly come across the Fubini polynomials (also known as the ordered Bell polynomials) $\mathcal{F}_{n}(x)=\sum_{k=1}^{n} k !\left\{\begin{array}{l}n \\ k\end{array}\right\} x^{k}$. 


\section{A decomposition theorem for web matrices}

The main result of this section is a theorem that explains how to form the web-colouring matrix of a web world that admits a decomposition as a disjoint union of at least two other web worlds. Physical arguments tell us that such webs do not contribute to the exponents of scattering amplitudes [9] and this is something we will discuss further and exploit in Section 4.

In order to be able to state our main theorem, we must first define some new numbers and a new product on formal power series.

Let $\left(\left(\left(\begin{array}{c}k \\ i_{1}, \ldots, i_{m}\end{array}\right)\right)\right)^{\star}$ be the number of $0-1$ fillings of an $m$ row and $k$ column array such that there are precisely $i_{1}$ ones on the top row, $i_{2}$ ones in the second row, etc., and there are no columns of only zeros.

Lemma 7. $\left(\left(\left(\begin{array}{c}k \\ i_{1}, \ldots, i_{m}\end{array}\right)\right)\right)^{\star}=\left[u_{1}^{i_{1}} u_{2}^{i_{2}} \cdots u_{m}^{i_{m}}\right]\left(\left(1+u_{1}\right) \cdots\left(1+u_{m}\right)-1\right)^{k}$.

Proof. The generating function for an entry in row $j$ of the $m \times k$ array is $u_{j}^{0}+u_{j}^{1}=1+u_{j}$. The only forbidden configuration in the array is a column of all zeros, so the generating function for columns is

$$
\left(\prod_{j=1}^{m}\left(u_{j}^{0}+u_{j}^{1}\right)\right)-u_{1}^{0} u_{2}^{0} \cdots u_{m}^{0}=\left(1+u_{1}\right) \cdots\left(1+u_{m}\right)-1 .
$$

Since there are no restrictions on rows, the generating function for the $m \times k$ array is therefore

$$
\left(\left(1+u_{1}\right) \cdots\left(1+u_{m}\right)-1\right)^{k} .
$$

The number of arrays with $i_{1}$ ones in the first row, $i_{2}$ ones in the second row, and so on, is the coefficient of $u_{1}^{i_{1}} u_{2}^{i_{2}} \cdots u_{m}^{i_{m}}$ in the above generating function:

$$
\left(\left(\left(\begin{array}{c}
k \\
i_{1}, \ldots, i_{m}
\end{array}\right)\right)\right)^{\star}=\left[u_{1}^{i_{1}} u_{2}^{i_{2}} \cdots u_{m}^{i_{m}}\right]\left(\left(1+u_{1}\right) \cdots\left(1+u_{m}\right)-1\right)^{k} .
$$

It is a simple exercise to check that $\left(\left(\left(\begin{array}{l}k \\ i_{1}\end{array}\right)\right)\right)^{\star}=\left(\begin{array}{c}k \\ i_{1}\end{array}\right)$ and $\left(\left(\left(\begin{array}{c}k \\ i_{1}, i_{2}\end{array}\right)\right)\right)^{\star}=\left(\begin{array}{c}k \\ k-i_{1}, k-i_{2}, i_{1}+i_{2}-k\end{array}\right)$. The general expression for $m=3$ is

$$
\left(\left(\left(\begin{array}{c}
k \\
i_{1}, i_{2}, i_{3}
\end{array}\right)\right)\right)^{\star}=\left(\begin{array}{c}
k \\
i_{1}
\end{array}\right) \sum_{a=0}^{i_{2}}\left(\begin{array}{c}
i_{1} \\
a
\end{array}\right)\left(\begin{array}{c}
k-i_{1} \\
i_{2}-a
\end{array}\right)\left(\begin{array}{c}
i_{1}+i_{2}-a \\
k-i_{3}
\end{array}\right) .
$$

We will now define a new product of power series that will both help in presenting our results concerning web worlds, but also serves in establishing a new automatic way to generate general combinatorial identities from web worlds. The black diamond product $\checkmark$ is a binary operation that is both commutative and associative, and we define it in a very general form since this is how we will typically be using it. 
Definition 8. Given $A^{(1)}(x), \ldots, A^{(m)}(x) \in \mathbb{C}[[x]]$ where $A^{(k)}(x)=\sum_{n \geqslant 0} a_{n}^{(k)} x^{n}$, we define the black diamond product of $A^{(1)}(x), \ldots, A^{(m)}(x)$ as:

$$
A^{(1)}(x) \diamond A^{(2)}(x) \diamond \cdots A^{(m)}(x)=\sum_{k \geqslant 0} x^{k} \sum_{i_{1} \geqslant 0} \cdots \sum_{i_{m} \geqslant 0} a_{i_{1}}^{(1)} \cdots a_{i_{m}}^{(m)}\left(\left(\left(\begin{array}{c}
k \\
i_{1}, \ldots, i_{m}
\end{array}\right)\right)\right)^{\star} .
$$

Let us note that if the power series $A^{(i)}(x)$ are each polynomials of degree $n_{i}$, then the product may be written in the more computationally efficient form:

$$
A^{(1)}(x) \diamond \cdots A^{(m)}(x)=\sum_{k=0}^{n_{1}+\cdots+n_{m}} x^{k} \sum_{\substack{i_{1} \in\left[0, n_{1}\right], \ldots, i_{m} \in\left[0, n_{m}\right] \\
i_{1} \vee \cdots \vee i_{m} \leqslant k \leqslant i_{1}+\cdots+i_{m}}} a_{i_{1}}^{(1)} \cdots a_{i_{m}}^{(m)}\left(\left(\left(\begin{array}{c}
k \\
i_{1}, \ldots, i_{m}
\end{array}\right)\right)\right)^{\star} .
$$

The unit of the black diamond product is 1, i.e. $1 \checkmark A(x)=A(x)$. To recap on our point before the definition, due to commutativity and associativity the order in which power series appear in the black diamond product does not change its outcome. In other words, given the power series $\left\{A^{(i)}(x)\right\}_{i \in[1, m]}$ and a permutation $\pi \in \mathcal{S}_{m}$,

$$
A^{(\pi(1))}(x) \diamond A^{(\pi(2))}(x) \diamond \cdots \diamond A^{(\pi(m))}=A^{(1)}(x) \diamond A^{(2)}(x) \diamond \cdots \diamond A^{(m)}(x) .
$$

We will abbreviate $\overbrace{A(x) \diamond \cdots \triangleleft A(x)}^{m}$ to $A(x){ }^{m}$ with the convention that $A(x)^{\bullet}=1$.

Example 9. Suppose that $A^{(1)}(x)=\cdots=A^{(m)}(x)=x$. Then

$$
x \overbrace{x \rightarrow \cdots}^{m}=\sum_{k \geqslant 0} x^{k}\left(\left(\left(\begin{array}{c}
k \\
1, \ldots, 1
\end{array}\right)\right)\right)^{\star}
$$

where there are $m$ ones on the right hand side. The value $\left(\left(\left(\begin{array}{c}k \\ 1, \ldots, 1\end{array}\right)\right)\right)^{\star}$ is the number of ways to fill a table of $k$ columns and $m$ rows with 0 s and 1 s such that there is exactly one 1 in every row and there are no columns of only 0s. This is simply another way to encode an ordered set partition of an $m$-set into $k$ sets (if $a$ is in the $i$ th set of such a sequence then the solitary 1 in row $a$ of the array will be in the $i$ th column). This number is $k !\left\{\begin{array}{c}m \\ k\end{array}\right\}$ and so for all $m \geqslant 1$,

$$
x x^{m}=\sum_{k=1}^{m} x^{k} k !\left\{\begin{array}{c}
m \\
k
\end{array}\right\}=\mathcal{F}_{m}(x)
$$

Example 10. Using the fact that $\left(\left(\left(\begin{array}{c}k \\ i_{1}, i_{2}\end{array}\right)\right)\right)^{\star}=\left(\begin{array}{c}k \\ k-i_{1}, k-i_{2}, i_{1}+i_{2}-k\end{array}\right)$ and applying Equation 2, we have

$$
\left(x+x^{2}\right) \diamond\left(x+x^{2}\right)=x+7 x^{2}+12 x^{3}+6 x^{4} .
$$


Several of the power series that arise from taking the black diamond product of simple power series expressions appear to correspond to known sequences/power series. This suggests the black diamond product may be an object worthy of study in its own right. Some examples of these include: $x x^{n}=n x^{n}+(n+1) x^{n+1}$, the coefficients in the power series $x^{n} x^{n+1}$ appear to be given by [17, A253283], and the sequence of coefficients of

$$
x^{n} \diamond x^{n}=\sum_{k=0}^{n}\left(\begin{array}{c}
n+k \\
k
\end{array}\right)\left(\begin{array}{l}
n \\
k
\end{array}\right) x^{n+k}
$$

is known to count several different structures (see [17, A063007]). (We omit the proof of this final observation since it is not immediately relevant to the paper's goal.)

We are now ready to state our main theorem.

Theorem 11. Let $W_{1}, \ldots, W_{m}$ be web worlds on pairwise disjoint peg sets. Suppose that $D_{i}, D_{i}^{\prime} \in W_{i}$ for all $i \in[1, m]$. Let $W=W_{1} \cup W_{2} \cup \cdots \cup W_{m}$ be a new web world which is the disjoint union of $W_{1}, \ldots, W_{m}$. The diagrams $D=D_{1} \oplus \cdots \oplus D_{m}$ and $D^{\prime}=D_{1}^{\prime} \oplus \cdots \oplus D_{m}^{\prime}$ are web diagrams in $W$. Then $\mathfrak{M}_{D, D^{\prime}}^{(W)}(x) \in \mathbb{C}[[x]]$ where

$$
\mathfrak{M}_{D, D^{\prime}}^{(W)}(x)=\mathfrak{M}_{D_{1}, D_{1}^{\prime}}^{\left(W_{1}\right)}(x) \diamond \cdots \diamond \mathfrak{M}_{D_{m}, D_{m}^{\prime}}^{\left(W_{m}\right)}(x) .
$$

Proof. Suppose that $\alpha_{1}$ is an $i_{1}$-colouring of $D_{1}$ which constructs $D_{1}^{\prime}$ (i.e. $\operatorname{Recon}\left(D_{1}, \alpha\right)=$ $\left.D_{1}^{\prime}\right)$ and that $\alpha_{2}$ is a $i_{2}$-colouring of $D_{2}$ that constructs $D_{2}^{\prime}$, and so on. An $i$-chain is a totally ordered set of $i$ elements. The number of ways to $k$-colour the diagram $D=D_{1} \oplus \cdots \oplus D_{m}$ so that it becomes $D^{\prime}$ is the number of ways to embed the $m$ chains $i_{1}$-chain, $i_{2}$-chain, $\ldots, i_{m}$ chain, which represent the ordered colourings of $D_{1}, \ldots, D_{m}$, respectively, into a $k$-chain.

The value of $k$ must be at least the length of the largest of the $m$ chains, and of course must be at most the sum of the lengths of all constituent $m$ chains, i.e. $k \in\left[\max \left(i_{1}, \ldots, i_{m}\right), i_{1}+\cdots+i_{m}\right]$. Furthermore, this embedding must be surjective, for otherwise at least one of the $k$-colours $\{1, \ldots, k\}$ would not have any corresponding colour in the $m$ constituent chains. One can recast this in the form of a tabular 0-1 filling problem where we have $m$ rows and $k$ columns whereby the column indices of the $i_{r}$ ones that appear in row $r$ of the table indicate the new colours that they take on in the $k$-colouring. The surjectivity condition translates into there being no columns of all zeros. The number of ways to do this is therefore $\left(\left(\left(\begin{array}{c}k \\ i_{1}, \ldots, i_{m}\end{array}\right)\right)\right)^{\star}$.

Suppose that the entries of the web-colouring matrices that correspond to the diagrams are

$$
\mathfrak{M}_{D_{i}, D_{i}^{\prime}}^{\left(W_{i}\right)}(x)=a_{1}^{(i)} x+\cdots+a_{n_{i}}^{(i)} x^{n_{i}}=: A^{(i)}(x)
$$

for all $i \in[1, m]$. Since there are $a_{i_{j}}^{(j)}$ ways to colour $D_{j}$ to produce $D_{j}^{\prime}$ for all $j \in[1, m]$, the factor $a_{i_{1}}^{(1)} \cdots a_{i_{m}}^{(m)}$ must be included. Therefore

$$
\mathfrak{M}_{D, D^{\prime}}^{(W)}(x)=\sum_{i_{1}=1}^{n_{1}} \cdots \sum_{i_{m}=1}^{n_{m}} a_{i_{1}}^{(1)} \cdots a_{i_{m}}^{(m)} \sum_{k=i_{1} \vee \cdots \vee i_{m}}^{i_{1}+\cdots+i_{m}} x^{k}\left(\left(\left(\begin{array}{c}
k \\
i_{1}, \ldots, i_{m}
\end{array}\right)\right)\right)^{\star}
$$




$$
=\mathfrak{M}_{D_{1}, D_{1}^{\prime}}^{\left(W_{1}\right)}(x) \diamond \cdots \diamond \mathfrak{M}_{D_{m}, D_{m}^{\prime}}^{\left(W_{m}\right)}(x)
$$

Example 12. For all $i \in[1, m]$, let $D_{i}=\{(2 i-1,2 i, 1,1)\}$ be the web diagram that consists of a single edge between pegs $2 i-1$ and $2 i$, and let $W_{i}$ be the web world that consists of the single web diagram $D_{i}$. Then $\mathfrak{M}^{\left(W_{i}\right)}(x)=(x)$, a $1 \times 1$ matrix. Since the conditions of Theorem 11 hold, the web-colouring matrix of $W=\left\{D=D_{1} \oplus \cdots \oplus D_{m}\right\}$, is

$$
\mathfrak{M}^{(W)}(x)=\left(\sum_{k=1}^{m} x^{k}\left(\left(\left(\begin{array}{c}
k \\
1, \ldots, 1
\end{array}\right)\right)\right)^{\star}\right)=\left(\mathcal{F}_{m}(x)\right),
$$

from Example 9.

Corollary 13. With the same setup as in Theorem 11,

$$
\text { trace } \mathfrak{M}^{(W)}(x)=\sum_{D_{1} \in W_{1}} \cdots \sum_{D_{m} \in W_{m}} \mathfrak{M}_{D_{1}, D_{1}}^{\left(W_{1}\right)}(x) \cdots \cdots \mathfrak{M}_{D_{m}, D_{m}}^{\left(W_{m}\right)}(x)
$$

\section{A method for generating combinatorial identities}

In this section we will outline a method for generating combinatorial identities by using the black diamond product in conjunction with a result concerning the trace of web-mixing matrices for disjoint web worlds.

Proposition 14. Let $W$ be a web world that is the disjoint union of at least two web worlds. Then all entries of the web-mixing matrix $\mathfrak{R}^{(W)}$ are zero, and consequently trace $\mathfrak{R}^{(W)}=0$.

A statistical physics-based proof of this result has been given in Gardi et al. [9, Section 5.1]. The essential physical idea, elaborated further in [11], is that exponents of scattering amplitudes can only contain interactions between non-disjoint groups of quarks and gluons.

The relationship between the web-mixing matrices and web-colouring matrices is

$$
\mathfrak{R}_{D, D^{\prime}}^{(W)}=\int_{-1}^{0} \frac{\mathfrak{M}_{D, D^{\prime}}^{(W)}(x)}{x} d x .
$$

Integrating formal power series can be a contentious issue, so let us be clear that the integrals we perform in this paper are definite integrals and always correspond to the transformation:

$$
a_{1} x+a_{2} x^{2}+a_{3} x^{3}+a_{4} x^{4}+\cdots \quad \mapsto \quad a_{1}-a_{2} / 2+a_{3} / 3-a_{4} / 4+\cdots .
$$

Suppose that $W_{1}, \ldots, W_{n}$ are web worlds on disjoint peg sets that all have the same web-colouring matrix $M=\mathfrak{M}^{\left(W_{i}\right)}(x)$. (This happens for example when all web worlds are 
equivalent to one another by relabeling the peg sets that they are defined upon.) Suppose further that the diagonal entries of $M$ are $\left(G_{1}(x), \ldots, G_{t}(x)\right)$ and that

$$
\left\{G_{1}(x), \ldots, G_{t}(x)\right\}=\left\{H_{1}(x), \ldots, H_{s}(x)\right\}
$$

where each of the $H_{i}(x)$ are distinct and have multiplicities $\left(h_{1}, \ldots, h_{s}\right)$ as diagonal entries in $M$. Then

$$
\begin{aligned}
& \operatorname{trace} \mathfrak{M}^{(W)}(x)=\sum_{D_{1} \in W_{1}} \cdots \sum_{D_{m} \in W_{m}} \mathfrak{M}_{D_{1}, D_{1}}^{\left(W_{1}\right)}(x) \diamond \cdots \mathfrak{M}_{D_{m}, D_{m}}^{\left(W_{m}\right)}(x) \\
& =\sum_{\left(i_{1}, \ldots, i_{m}\right) \in[1, t]^{m}} G_{i_{1}}(x) \diamond \cdots \diamond G_{i_{m}}(x) \\
& =\sum_{\left(j_{1}, \ldots, j_{m}\right) \in[1, s]^{m}} h_{j_{1}} \cdots h_{j_{m}} H_{j_{1}}(x) \diamond \cdots \diamond H_{j_{m}}(x) \\
& =\sum_{\substack{a_{1}, \ldots, a_{s} \geqslant 0 \\
a_{1}+\cdots+a_{s}=m}} h_{1}^{a_{1}} \cdots h_{s}^{a_{s}}\left(\begin{array}{c}
m \\
a_{1}, \ldots, a_{s}
\end{array}\right) H_{1}(x)^{a_{1}} \diamond \cdots \diamond H_{s}(x)^{a_{s}} .
\end{aligned}
$$

For the case that there are only two different power series that appear as diagonal entries of $M$, we have

$$
\text { trace } \mathfrak{M}^{(W)}(x)=\sum_{a=0}^{m} h_{1}^{a} h_{2}^{m-a}\left(\begin{array}{c}
m \\
a
\end{array}\right) H_{1}(x) \bullet H_{2}(x)^{\bullet-a} .
$$

Theorem 15. Let $W$ be a web world whose web-colouring matrix has s different diagonal entries $\left(H_{1}(x), \ldots, H_{s}(x)\right)$ that appear with multiplicities $\left(h_{1}, \ldots, h_{s}\right)$. Then for all positive integers $m$, we have

$$
\sum_{\substack{a_{1}, \ldots, a_{s} \geqslant 0 \\
a_{1}+\cdots+a_{s}=m}} h_{1}^{a_{1}} \cdots h_{s}^{a_{s}}\left(\begin{array}{c}
m \\
a_{1}, \ldots, a_{s}
\end{array}\right) \int_{-1}^{0} H_{1}(x) \diamond a_{1} \diamond \cdots H_{s}(x) a_{s} \frac{d x}{x}=0 .
$$

The expression for the $s=2$ case is:

$$
\sum_{a=0}^{m} h_{1}^{a} h_{2}^{m-a}\left(\begin{array}{c}
m \\
a
\end{array}\right) \int_{-1}^{0} H_{1}(x){ }^{a} \diamond H_{2}(x) \diamond m-a \frac{d x}{x}=0 .
$$

We will apply the above theorem to two extremely simple web worlds to see the combinatorial identities that emerge.

Example 16. Let $W$ be one of the web worlds of Example 12 so that $\mathfrak{M}^{(W)}(x)=(x)$, a $1 \times 1$ matrix. Applying Theorem 15 we have $s=h_{1}=1$ and $H_{1}(x)=x$. From Equation 4 we have

$$
H_{1}(x)^{\star m}=\sum_{k=1}^{m} x^{k} k !\left\{\begin{array}{c}
m \\
k
\end{array}\right\}
$$


and so

$$
\begin{aligned}
\int_{-1}^{0} H_{1}(x) \frac{m}{x} \frac{d x}{x} & =\left[\sum_{k=1}^{m} \frac{x^{k}}{k} k !\left\{\begin{array}{c}
m \\
k
\end{array}\right\}\right]_{-1}^{0} \\
& =\sum_{k=1}^{m}(-1)^{k+1}(k-1) !\left\{\begin{array}{c}
m \\
k
\end{array}\right\} .
\end{aligned}
$$

This gives us the identity:

$$
\sum_{a_{1}=m} 1^{a_{1}}\left(\begin{array}{c}
m \\
a_{1}
\end{array}\right) \int_{-1}^{0} H_{1}(x) \cdot m \frac{d x}{x}=\sum_{k=1}^{m}(-1)^{k+1}(k-1) !\left\{\begin{array}{l}
m \\
k
\end{array}\right\}=0 .
$$

This identity can also be found in [21, Eqn. 27].

Example 17. Let $W$ be the web world of Example 4, with

$$
\mathfrak{M}^{(W)}(x)=\left(\begin{array}{cc}
x & 2 x^{2} \\
0 & x+2 x^{2}
\end{array}\right) \text {. }
$$

In this case $s=2, H_{1}(x)=x, H_{2}(x)=x+2 x^{2}$, and $h_{1}=h_{2}=1$. From Equation 4 , we have $H_{1}(x)^{m}=\mathcal{F}_{m}(x)$. One can show that $H_{2}(x)^{m}=\mathcal{F}_{2 m}(x)$ and then applying Theorem 15 we have

$$
\begin{aligned}
H_{1}(x) 、 H_{2}(x) \bullet-a & =\sum_{k=0}^{2 m-a} x^{k} \sum_{i_{1}, i_{2}} i_{1} !\left\{\begin{array}{l}
a \\
i_{1}
\end{array}\right\} i_{2} !\left\{\begin{array}{c}
2 m-2 a \\
i_{2}
\end{array}\right\}\left(\left(\left(\begin{array}{c}
k \\
i_{1}, i_{2}
\end{array}\right)\right)\right)^{\star} \\
& =\sum_{k=0}^{2 m-a} x^{k} \sum_{i_{1}, i_{2}} i_{1} !\left\{\begin{array}{c}
a \\
i_{1}
\end{array}\right\} i_{2} !\left\{\begin{array}{c}
2 m-2 a \\
i_{2}
\end{array}\right\}\left(\begin{array}{c}
k \\
k-i_{1}, k-i_{2}, i_{1}+i_{2}-k
\end{array}\right) .
\end{aligned}
$$

This gives us the identity

$$
\sum_{a=0}^{m} \sum_{k=1}^{2 m-a} \sum_{i_{1}, i_{2}}\left(\begin{array}{c}
m \\
a
\end{array}\right) \frac{(-1)^{k+1}}{k} i_{1} ! i_{2} !\left\{\begin{array}{l}
a \\
i_{1}
\end{array}\right\}\left\{\begin{array}{c}
2 m-2 a \\
i_{2}
\end{array}\right\}\left(\begin{array}{c}
k \\
k-i_{1}, k-i_{2}, i_{1}+i_{2}-k
\end{array}\right)=0 .
$$

We do not know if this is a known identity.

\section{$5 \quad$ Repeated entries in web matrices}

One observation that is apparent when calculating the web matrices (be they web-mixing or web-colouring) of web worlds is that there are a large number of entries that are the same. A deeper understanding of this property would simplify the calculation of webmixing matrices, and has even led, for certain families of web diagram, to obtaining the web-mixing matrix for arbitrary numbers of gluons [4]. In this section we prove two new 
results to explain some of these repetitive entries. The first theorem gives one explanation for repeated entries found on the diagonal of a web-colouring matrix, and also explains the same for the web-mixing matrix since the entries of the latter are a simple integral transformation of those in the web-colouring matrix. This theorem also builds on our earlier result [5, Thm. 3.4].

Given a poset $P=(P, \prec)$, its comparability graph $\operatorname{comp}(P)$ is the graph whose vertices are the elements of $P$, with $x, y \in P$ adjacent in $\operatorname{comp}(P)$ if $x \prec y$ or $y \prec x$.

Theorem 18. Let $D$ and $D^{\prime}$ be web diagrams in a web world $W$ with

$$
D=E_{1} \oplus \cdots \oplus E_{k} \quad \text { and } \quad D^{\prime}=E_{1}^{\prime} \oplus \cdots \oplus E_{k^{\prime}}^{\prime}
$$

where each of the constituent diagrams $E_{i}$ and $E_{i}^{\prime}$ are indecomposable. Suppose that the members of $\left(E_{1}, \ldots, E_{k}\right)$ are distinct and the members of $\left(E_{1}^{\prime}, \ldots, E_{k^{\prime}}^{\prime}\right)$ are also distinct. If $\operatorname{comp}(P(D))=\operatorname{comp}\left(P\left(D^{\prime}\right)\right)$, then $\mathfrak{M}_{D, D}^{(W)}(x)=\mathfrak{M}_{D^{\prime}, D^{\prime}}^{(W)}(x)$.

Proof. First let us consider the web diagram $D=E_{1} \oplus \cdots \oplus E_{k}$ where each of the diagrams $E_{i}$ is indecomposable. All $\ell$-colourings $\alpha$ such that $\operatorname{Recon}(D, \alpha)=D$ must have the property that $\alpha(e)=\alpha\left(e^{\prime}\right)$ whenever $e, e^{\prime} \in E_{i}$ because $E_{i}$ is indecomposable. Thus $\alpha$ must be a surjective map from $\left\{E_{1}, \ldots, E_{k}\right\}$ to $[1, \ell]$. Equivalently $\alpha$ surjectively maps the $k$ elements of the decomposition poset $P(D)$ to the total order on $[1, \ell]$. Let us call the number of these surjective maps $\Theta(P(D), \ell)$. This number is related to the number $\Omega(P(D), \ell)$ of order preserving maps from the poset $P(D)$ to $[1, \ell]$ via an inclusionexclusion argument:

$$
\Omega(P(D), \ell)=\sum_{k}\left(\begin{array}{l}
\ell \\
k
\end{array}\right)(-1)^{\ell-k} \Omega(P(D), \ell) .
$$

(See [5, Lemma 3.3] for further details.) Using this we have

$$
\mathfrak{M}_{D, D}^{(W)}(x)=\sum_{\ell \geqslant 0} \Theta(P(D), \ell) x^{\ell}=\sum_{\ell \geqslant 0} x^{\ell} \sum_{k}\left(\begin{array}{l}
\ell \\
k
\end{array}\right) \Omega(P(D), k) .
$$

The same is true for $D^{\prime}$, so we have

$$
\mathfrak{M}_{D^{\prime}, D^{\prime}}^{(W)}(x)=\sum_{\ell \geqslant 0} x^{\ell} \sum_{k}\left(\begin{array}{l}
\ell \\
k
\end{array}\right) \Omega\left(P\left(D^{\prime}\right), k\right) .
$$

If $\operatorname{comp}(P(D))=\operatorname{comp}\left(P\left(D^{\prime}\right)\right)$ then by Stanley [18, Cor. 4.4] we have that $\Omega(P(D))=$ $\Omega\left(P\left(D^{\prime}\right)\right)$, and consequently the expressions in Equations 10 and 11 are the same.

It is not immediately obvious that the previous theorem does indeed help with enlarging the collection of diagrams that are known to have the same diagonal entries in a web-colouring matrix. We give here a non-trivial example, and one which cannot be discovered by our subsequent Theorem 22 . 

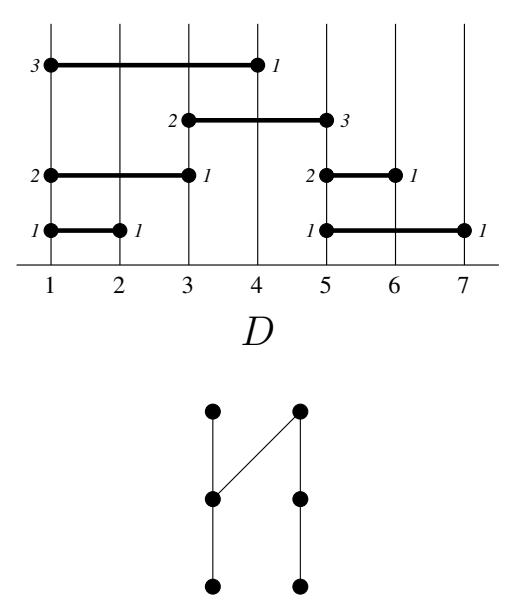

$P(D)$
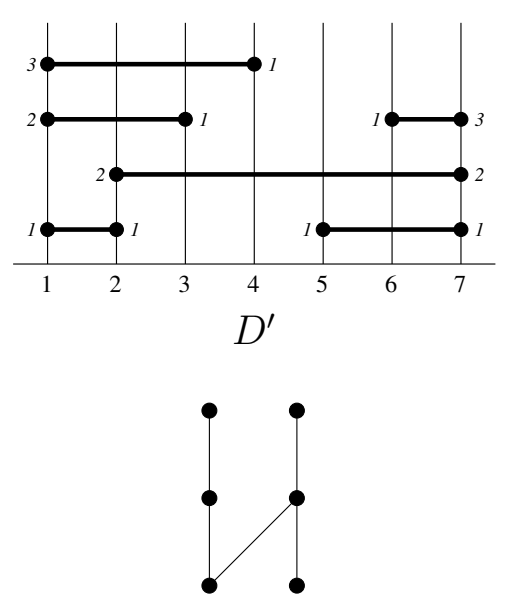

$P\left(D^{\prime}\right)$

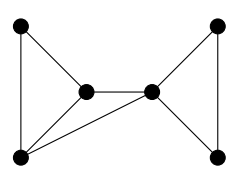

$G$

Figure 5: The web diagrams, their deconstruction posets, and web graph for Example 19.

Example 19. Let $D=\{(1,2,1,1),(1,3,2,1),(1,4,3,1),(3,5,2,3),(5,6,2,1),(5,7,1,1)\}$ and $D^{\prime}=\{(1,2,1,1),(1,3,2,1),(1,4,3,1),(2,7,2,3),(6,7,1,2),(5,7,1,1)\}$. The Hasse diagrams for $P(D)$ and $P\left(D^{\prime}\right)$ are illustrated in Figure 5 and are clearly different. However we have that $\operatorname{comp}(P(D))=\operatorname{comp}\left(P\left(D^{\prime}\right)\right)=G$ and we can conclude that $\mathfrak{M}_{D, D}^{(W)}(x)=$ $\mathfrak{M}_{D^{\prime}, D^{\prime}}^{(W)}(x)$.

Our second result addresses general entries of the web-colouring matrix and upsidedown web diagrams. Given a web diagram $D$, let flip $(D)$ be the web diagram achieved by turning it upside-down. This operation is, by definition, an involution.

Definition 20. Let $D=\left\{e_{i}=\left(a_{i}, b_{i}, c_{i}, d_{i}\right): 1 \leqslant i \leqslant m\right\}$ be a web diagram in a web world $W$. The flip of $D$, flip $(D)$ is the web diagram in $W$ with edges:

$$
\text { flip }(D)=\left\{\left(a_{i}, b_{i}, p_{a_{i}}+1-c_{i}, p_{b_{i}}+1-d_{i}\right): 1 \leqslant i \leqslant m\right\} .
$$

Example 21. Let $D$ be the web diagram in Example 19 and illustrated in Figure 5. Then

$$
f \operatorname{lip}(D)=\{(1,2,3,1),(1,3,2,2),(1,4,3,1),(3,5,1,1),(5,6,2,1),(5,7,3,1)\} .
$$




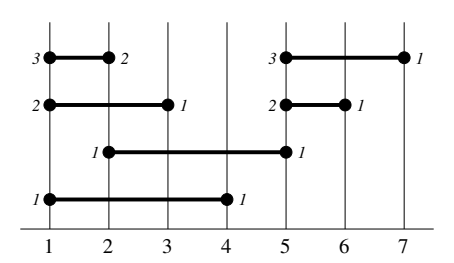

flip $(D)$

Theorem 22. Let $W$ be a web world and $D, D^{\prime} \in W$. Then $\mathfrak{M}_{D, D^{\prime}}^{(W)}(x)=\mathfrak{M}_{\text {flip }(D) \text {,flip }\left(D^{\prime}\right)}^{(W)}(x)$.

Proof. Let $W$ be a web world and $D, D^{\prime}$ diagrams in $W$. To prove the theorem it suffices to show that for every $k$-colouring $\alpha$ of $D$ that yields $D^{\prime}$, there is a unique $k$-colouring $\beta$ of flip $(D)$ that yields flip $\left(D^{\prime}\right)$. Define the colouring $\beta$ as follows: if $e=(a, b, x, y) \in D$ with $\alpha(e)=\ell$, then let $\beta(e)=k+1-\ell$.

We will now show that $\operatorname{Recon}(f \operatorname{lip}(D), \beta)=f \operatorname{lip}\left(D^{\prime}\right)$ which implies the result. Notice that in general if $B_{1}, \ldots, B_{m}$ are web diagrams then

$$
\operatorname{flip}\left(B_{1} \oplus \cdots \oplus B_{m}\right)=\operatorname{flip}\left(B_{m}\right) \oplus \cdots \oplus \operatorname{flip}\left(B_{1}\right) .
$$

We have:

$$
\begin{aligned}
& \operatorname{Recon}(f \operatorname{lip}(D), \beta)=\operatorname{rel}\left(f \operatorname{lip}(D)_{\beta}(1)\right) \oplus \cdots \oplus \operatorname{rel}\left(\operatorname{flip}(D)_{\beta}(k)\right) \\
& =\mathrm{flip}\left(\operatorname{rel}\left(D_{\beta}(1)\right)\right) \oplus \cdots \oplus \operatorname{flip}\left(\operatorname{rel}\left(D_{\beta}(k)\right)\right) \text {. }
\end{aligned}
$$

Using Equation 12, and applying flip to both sides of the previous equation, we have

$$
\begin{aligned}
& f \operatorname{lip}(\operatorname{Recon}(f \operatorname{lip}(D), \beta))=\operatorname{flip}\left(f \operatorname{lip}\left(\operatorname{rel}\left(D_{\beta}(1)\right)\right) \oplus \cdots \oplus \operatorname{flip}\left(\operatorname{rel}\left(D_{\beta}(k)\right)\right)\right) \\
& =\operatorname{flip}\left(f l i p\left(\operatorname{rel}\left(D_{\beta}(k)\right)\right)\right) \oplus \cdots \oplus \operatorname{flip}\left(f \operatorname{lip}\left(\operatorname{rel}\left(D_{\beta}(1)\right)\right)\right) \\
& =\operatorname{rel}\left(D_{\beta}(k)\right) \oplus \cdots \oplus \operatorname{rel}\left(D_{\beta}(1)\right) \text {. }
\end{aligned}
$$

Since those edges of $D$ that are coloured $\ell$ using the colouring $\beta$ are precisely the same as the edges that are coloured $k+1-\ell$ using the colouring $\alpha$, we have $D_{\beta}(\ell)=D_{\alpha}(k+1-\ell)$ and

$$
\begin{aligned}
& \text { flip }(\operatorname{Recon}(f \operatorname{lip}(D), \beta))=\operatorname{rel}\left(D_{\alpha}(1)\right) \oplus \cdots \oplus \operatorname{rel}\left(D_{\alpha}(k)\right) \\
& =D^{\prime} \text {. }
\end{aligned}
$$

Applying flip to both sides gives $\operatorname{Recon}(f \operatorname{lip}(D), \beta)=\mathrm{flip}\left(D^{\prime}\right)$, as was required.

\section{The squares of web-colouring and web-mixing matrices}

In this section we show how to calculate the entries for the square of a web-colouring matrix and give the first mathematical proof that web-mixing matrices are idempotent. This property has been established using physical arguments by Gardi and White [12, $\S 3]$, and plays an important role in QCD calculations. Idempotence implies, for example, that all web-mixing matrices have eigenvalues zero or one (with some multiplicity). Only combinations of web diagrams in a given web world associated with unit eigenvalues of the mixing matrix survive in the exponent of the scattering amplitude. 


\begin{tabular}{|c|c|}
\hline$n$ & $L_{n}(x)$ \\
\hline \hline 0 & 1 \\
1 & $x^{2}$ \\
2 & $2 x^{3}+2 x^{4}$ \\
3 & $6 x^{4}+12 x^{5}+6 x^{6}$ \\
4 & $x^{4}+26 x^{5}+73 x^{6}+72 x^{7}+24 x^{8}$ \\
5 & $12 x^{5}+156 x^{6}+516 x^{7}+732 x^{8}+480 x^{9}+120 x^{10}$ \\
6 & $2 x^{5}+126 x^{6}+1206 x^{7}+4322 x^{8}+7680 x^{9}+7320 x^{10}+3600 x^{11}+720 x^{12}$ \\
\hline
\end{tabular}

Figure 6: The first few power series $L_{n}(x)$.

Theorem 23. Let $W$ be a web world whose diagrams each have $n$ edges. Let $D, D^{\prime} \in W$ and suppose that $\mathfrak{M}_{D, D^{\prime}}^{(W)}(x)=z_{1} x+\cdots+z_{n} x^{n}$. Then

$$
\left(\mathfrak{M}^{(W)}(x)\right)_{D, D^{\prime}}^{2}=\sum_{i=1}^{n} z_{i} L_{i}(x)
$$

where $L_{i}(x)=\sum_{j, k \geqslant 1} x^{j+k} \sum_{b=0}^{j} \sum_{a=0}^{k}(-1)^{j+k-(b+a)}\left(\begin{array}{l}j \\ b\end{array}\right)\left(\begin{array}{c}k \\ a\end{array}\right)\left(\begin{array}{c}a b \\ i\end{array}\right)$. (See Figure 6.)

Figure 6 presents the expressions for $L_{n}(x)$ for all $n \in[1,7]$. The previous theorem may be summarised by saying that the square of the web-colouring matrix $\mathfrak{M}^{(W)}(x)^{2}$ is the image of $\mathfrak{M}^{(W)}(x)$ under the linear operator $T: \mathbb{C}[[x]] \rightarrow \mathbb{C}[[x]]$ which maps the basis $\left(x^{i}\right)_{i \geqslant 0}$ for $\mathbb{C}[[x]]$ to $\left(L_{i}(x)\right)_{i \geqslant 0}$.

Proof. Let $\alpha \in \operatorname{Colours}(W)$. Given two colourings $\alpha, \alpha^{\prime} \in \operatorname{Colours}(W)$ there is a unique $\gamma \in \operatorname{Colours}(W)$ such that $\operatorname{Recon}(D, \gamma)=\operatorname{Recon}(\operatorname{Recon}(D, \alpha), \beta)$. In such an instance we write $\gamma \equiv \beta \circ \alpha$. Recall that the $\left(D, D^{\prime}\right)$ entry of the web-colouring matrix of $W$ is:

$$
\mathfrak{M}_{D, D^{\prime}}^{(W)}(x)=\sum_{\substack{\alpha \in \operatorname{Colours}(W) \\ \operatorname{Recon}(D, \alpha)=D^{\prime}}} x^{|\alpha|}
$$

and suppose that this is $\mathfrak{M}_{D, D^{\prime}}^{(W)}(x)=z_{1} x+\cdots+z_{n} x^{n}$. Then

$$
\begin{aligned}
\left(\mathfrak{M}^{(W)}(x)\right)_{D, D^{\prime}}^{2} & =\sum_{D^{\prime \prime} \in W} \mathfrak{M}_{D, D^{\prime \prime}}^{(W)}(x) \mathfrak{M}_{D^{\prime \prime}, D^{\prime}}^{(W)}(x) \\
& =\sum_{D^{\prime \prime} \in W} \sum_{\substack{\alpha \in \operatorname{Colours}(W) \\
\operatorname{Recon}(D, \alpha)=D^{\prime \prime}}} x^{|\alpha|} \sum_{\substack{\beta \in \operatorname{Colours}(W) \\
\operatorname{Recon}\left(D^{\prime \prime}, \beta\right)=D^{\prime}}} x^{|\beta|} \\
& =\sum_{j, k=1} \sum_{\begin{array}{c}
\alpha \in \operatorname{Colours}_{j}(W) \\
\beta \in \operatorname{Colours}_{k}(W) \\
\operatorname{Recon}\left(\operatorname{Recon}_{(D, \alpha), \beta)=D^{\prime}}\right.
\end{array}} x^{j+k}
\end{aligned}
$$




$$
\begin{aligned}
& =\sum_{j, k=1}^{n} \sum_{i=1}^{n} \sum_{\substack{\gamma \in \operatorname{Colours~}_{i}(W) \\
\text { Recon }(D, \gamma)=D^{\prime}}} \sum_{\substack{\alpha \in \operatorname{Colours}_{j}(W) \\
\beta \in \operatorname{Colours}_{k}(W) \\
\gamma \equiv \beta \circ \alpha}} x^{j+k} \\
& =\sum_{i=1}^{n} \sum_{\substack{\gamma \in \operatorname{Colours}_{i}(W), \operatorname{Recon}(D, \gamma)=D^{\prime}}} \sum_{j, k=1}^{n} N_{j, k}(i) x^{j+k}=\sum_{i=1}^{n} z_{i} \sum_{j, k=1}^{n} N_{j, k}(i) x^{j+k},
\end{aligned}
$$

where $N_{j, k}(i)$ is the number of pairs $(\beta, \alpha)$ of colourings in $\operatorname{Colours}_{j}(W) \times \operatorname{Colours}_{k}(W)$ such that $\beta \circ \alpha$ is equivalent to a $i$-colouring $\gamma \in \operatorname{Colours}_{i}(W)$. To see how a colouring of a colouring of a diagram can be turned into one colouring ${ }^{3}$, we consider the ordered pairs of colours. The diagram $D$ is coloured with $\alpha$ and reconstructed to give the diagram $D^{\prime \prime}$. The diagram $D^{\prime \prime}$ is coloured with $\beta$ and reconstructed to give the diagram $D^{\prime}$.

Let us consider what happens to an edge $e_{i}=\left(a_{i}, b_{i}, x_{i}, y_{i}\right) \in D$ during this process. In the first colouring, $\alpha\left(e_{i}\right)=v$, say, and this edge in $D^{\prime \prime}$ will be represented as $e_{i}^{\prime}=$ $\left(a_{i}, b_{i}, x_{i}^{\prime}, y_{i}^{\prime}\right)$ where $x_{i}^{\prime}$ and $y_{i}^{\prime}$ are the new heights of the endpoints of that edge in $D^{\prime \prime}$. The edge $e_{i}^{\prime}$ is now coloured $\beta\left(e_{i}^{\prime}\right)=u$, say, and this edge will be represented as $e_{i}^{\prime \prime}=$ $\left(a_{i}, b_{i}, x_{i}^{\prime \prime}, y_{i}^{\prime \prime}\right)$ in $D^{\prime}$ where $x_{i}^{\prime \prime}$ and $y_{i}^{\prime \prime}$ are the heights of the endpoints of $e_{i}^{\prime \prime}$ in $D^{\prime}$. Although the heights change from one diagram to the next, we can still index the edge in question throughout as the $i$ th edge $e_{i}$ and write $\alpha\left(e_{i}\right)=v$ and $\beta\left(e_{i}\right)=u$.

Let $D \in W$ and $(\alpha, \beta) \in \operatorname{Colours}_{j}(W) \times \operatorname{Colours}_{k}(W)$. Let us define

$$
D_{\beta, \alpha}(u, v):=\{e \in D: \beta(e)=u \text { and } \alpha(e)=v\} .
$$

Then

$$
D^{\prime}=\operatorname{rel}\left(D_{\beta, \alpha}(1,1)\right) \oplus \cdots \oplus \operatorname{rel}\left(D_{\beta, \alpha}(1, k)\right) \oplus \cdots \oplus \operatorname{rel}\left(D_{\beta, \alpha}(j, 1)\right) \oplus \cdots \oplus \operatorname{rel}\left(D_{\beta, \alpha}(j, k)\right) .
$$

In other words, the subweb-diagram of $\operatorname{Recon}(D, \alpha)$ whose edges $e$ have colour $\beta(e)=1$ will maintain their order relative to one another in $D^{\prime}$ and be the lowest. But amongst these edges, which will be the lowest w.r.t. the colouring $\alpha$ of $D$ ? It will be those edges $e \in D$ that are coloured $\alpha(e)=1$, followed by all those edges $e \in D$ with $\alpha(e)=2$, and so on. Thus the lexicographic order on all such pairs of colourings that exists corresponds to the total order for a single colouring.

For example, if we have the pairs of colourings $(\beta(e), \alpha(e))=(1,3),(1,5),(2,1),(2,2)$, $(2,5),(3,4)$, then these correspond to the colours $\gamma(e)=1,2,3,4,5,6$, respectively.

The number $N_{j, k}(i)$ is therefore the number of $j \times k$ zero-one matrices that have exactly $i$ ones and there are neither columns nor rows of only zeros. This number is known to be (see $[3,2]$ or [15, Eqn. 120]):

$$
N_{m, n}(r)=\sum_{\ell \geqslant r} \sum_{d \mid \ell}(-1)^{n+m-(d+\ell / d)}\left(\begin{array}{c}
m \\
d
\end{array}\right)\left(\begin{array}{c}
n \\
\ell / d
\end{array}\right)\left(\begin{array}{l}
\ell \\
r
\end{array}\right)
$$

\footnotetext{
${ }^{3}$ The argument used here is reminiscent of the double application of the statistical physics-based replica trick in ref. [12].
} 


$$
=\sum_{b=0}^{m} \sum_{a=0}^{n}(-1)^{n+m-(b+a)}\left(\begin{array}{c}
m \\
b
\end{array}\right)\left(\begin{array}{l}
n \\
a
\end{array}\right)\left(\begin{array}{c}
a b \\
r
\end{array}\right)
$$

Therefore $\left(\mathfrak{M}^{(W)}(x)\right)_{D, D^{\prime}}^{2}=\sum_{i=1}^{n} z_{i} L_{i}(x)$ where

$$
L_{i}(x)=\sum_{j, k \geqslant 1} x^{j+k} \sum_{\substack{a \in[0, k] \\
b \in[0, j]}}(-1)^{j+k-(b+a)}\left(\begin{array}{l}
j \\
b
\end{array}\right)\left(\begin{array}{l}
k \\
a
\end{array}\right)\left(\begin{array}{c}
a b \\
i
\end{array}\right) .
$$

As mentioned above, the following theorem has already been proven using a physics argument (see [12, Sect. 3]). Here we give the first combinatorial proof.

Theorem 24. Let $W$ be a web world whose web diagrams each have $n$ edges. The webmixing matrix $\mathfrak{R}^{(W)}$ is idempotent; $\left(\mathfrak{R}^{(W)}\right)^{2}=\mathfrak{R}^{(W)}$.

Proof. Using the same method of summation as we did at the start of the proof of Theorem 23,

$$
\begin{aligned}
& \left(\mathfrak{R}^{(W)}\right)_{D, D^{\prime}}^{2}=\sum_{D^{\prime \prime} \in W} \mathfrak{R}_{D, D^{\prime \prime}}^{(W)} \mathfrak{R}_{D^{\prime \prime}, D^{\prime}}^{(W)} \\
& =\sum_{i, j=1}^{n} \sum_{\substack{\alpha \in \operatorname{Colours}_{i}(W) \\
\beta \in \operatorname{Colours}_{j}(W) \\
\operatorname{Recon}(\operatorname{Recon}(D, \alpha), \beta)=D^{\prime}}} \frac{(-1)^{i-1}}{i} \frac{(-1)^{j-1}}{j} \\
& =\sum_{i, j=1}^{n} \frac{(-1)^{i-1}}{i} \frac{(-1)^{j-1}}{j} \sum_{\substack{\alpha \in \operatorname{Colours}_{i}(W) \\
\beta \in \operatorname{Colours}_{j}(W) \\
\operatorname{Recon}(\operatorname{Recon}(D, \alpha), \beta)=D^{\prime}}} 1 .
\end{aligned}
$$

Since

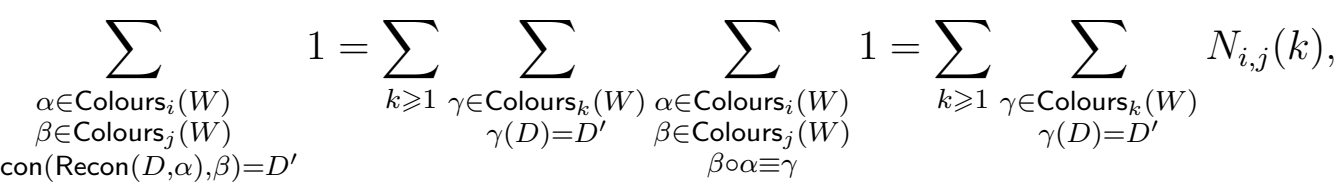

this gives

$$
\begin{aligned}
\left(\mathfrak{R}^{(W)}\right)_{D, D^{\prime}}^{2} & =\sum_{i, j=1}^{n} \frac{(-1)^{i-1}}{i} \frac{(-1)^{j-1}}{j} \sum_{k \geqslant 1} \sum_{\substack{\gamma \in \text { Colours }_{k}(W) \\
\gamma(D)=D^{\prime}}} N_{i, j}(k) \\
& =\sum_{k=1}^{n} \sum_{\substack{\gamma \in \operatorname{Colours}_{k}(W) \\
\gamma(D)=D^{\prime}}}(-1)^{-2} \sum_{i, j=1}^{n} \frac{(-1)^{i+j}}{i j} N_{i, j}(k) .
\end{aligned}
$$


The inner sum is

$$
\begin{aligned}
\sum_{i, j=1}^{n} \frac{(-1)^{i+j}}{i j} N_{i, j}(k) & =\sum_{i, j=1}^{n} \frac{(-1)^{i+j}}{i j} \sum_{a=0}^{i} \sum_{b=0}^{j}(-1)^{i+j-(a+b)}\left(\begin{array}{c}
i \\
a
\end{array}\right)\left(\begin{array}{l}
j \\
b
\end{array}\right)\left(\begin{array}{c}
a b \\
k
\end{array}\right) \\
& =\sum_{i, j=1}^{n} \sum_{a=0}^{i} \sum_{b=0}^{j}(-1)^{2(i+j)-(a+b)} \frac{1}{i}\left(\begin{array}{c}
i \\
a
\end{array}\right) \frac{1}{j}\left(\begin{array}{c}
j \\
b
\end{array}\right)\left(\begin{array}{c}
a b \\
k
\end{array}\right) \\
& =\sum_{i, j=1}^{n} \sum_{a=0}^{i} \sum_{b=0}^{j}(-1)^{a+b} \frac{1}{k}\left(\begin{array}{c}
i-1 \\
a-1
\end{array}\right)\left(\begin{array}{c}
j-1 \\
b-1
\end{array}\right)\left(\begin{array}{c}
a b-1 \\
k-1
\end{array}\right) \\
& =\frac{1}{k} \sum_{a, b=1}^{n}(-1)^{a+b}\left(\begin{array}{c}
a b-1 \\
k-1
\end{array}\right) \sum_{i=a}^{n} \sum_{j=b}^{n}\left(\begin{array}{c}
i-1 \\
a-1
\end{array}\right)\left(\begin{array}{c}
j-1 \\
b-1
\end{array}\right) \\
& =\frac{1}{k} \sum_{a, b=1}^{n}(-1)^{a+b}\left(\begin{array}{c}
a b-1 \\
k-1
\end{array}\right)\left(\begin{array}{c}
n \\
a
\end{array}\right)\left(\begin{array}{c}
n \\
b
\end{array}\right) .
\end{aligned}
$$

We now need to prove that for $1 \leqslant k \leqslant n$,

$$
F(n, k):=\sum_{a, b=1}^{n}(-1)^{a+b}\left(\begin{array}{c}
a b-1 \\
k-1
\end{array}\right)\left(\begin{array}{l}
n \\
a
\end{array}\right)\left(\begin{array}{l}
n \\
b
\end{array}\right)=(-1)^{k-1} .
$$

Let $G(n, k, a)=\sum_{b=1}^{n}(-1)^{b}\left(\begin{array}{c}n \\ b\end{array}\right)\left(\begin{array}{c}a b-1 \\ k-1\end{array}\right)$ so that $F(n, k)=\sum_{a=1}^{n}(-1)^{a}\left(\begin{array}{l}n \\ a\end{array}\right) G(n, k, a)$. Now

$$
\begin{aligned}
\left(\begin{array}{c}
a b-1 \\
k-1
\end{array}\right) & =\frac{(a b)(a b-1) \cdots(a b-k+1)}{a b(k-1) !} \\
& =\frac{1}{(k-1) !} \sum_{j=1}^{k}\left[\begin{array}{c}
k \\
j
\end{array}\right](-1)^{k-j}(a b)^{j-1},
\end{aligned}
$$

the second equality comes from Graham et al. [14, eqn. (6.13)] and $\left[\begin{array}{l}k \\ j\end{array}\right]$ are the Stirling numbers of the first kind. Replacing this into the expression

$$
\begin{aligned}
G(n, k, a) & =\sum_{b=1}^{n}(-1)^{b}\left(\begin{array}{l}
n \\
b
\end{array}\right) \frac{1}{(k-1) !} \sum_{j=1}^{k}\left[\begin{array}{l}
k \\
j
\end{array}\right](-1)^{k-j}(a b)^{j-1} \\
& =\frac{1}{(k-1) !} \sum_{j=1}^{k}\left[\begin{array}{l}
k \\
j
\end{array}\right](-1)^{k-j} a^{j-1} \sum_{b=1}^{n}(-1)^{b}\left(\begin{array}{l}
n \\
b
\end{array}\right) b^{j-1} .
\end{aligned}
$$

Using Graham et al. [14, Eqn. (6.19)]: $m !\left\{\begin{array}{c}n \\ m\end{array}\right\}=\sum_{k}\left(\begin{array}{c}m \\ k\end{array}\right) k^{n}(-1)^{m-k}$ where $\left\{\begin{array}{c}n \\ m\end{array}\right\}$ are the Stirling numbers of the second kind. The inner sum in the previous expression is

$$
\sum_{b=1}^{n}(-1)^{b}\left(\begin{array}{l}
n \\
b
\end{array}\right) b^{j-1}=n !\left\{\begin{array}{c}
j-1 \\
n
\end{array}\right\}(-1)^{n}-0^{j-1} .
$$


Since $k \in[1, n]$, and $j \leqslant k$, the Stirling number on the right hand side of the previous equation will always be zero for the values that we are summing over, and the only term that will contribute will be $-0^{j-1}$. This term itself will always be zero, except for when $j-1=0$. This gives

$$
\begin{aligned}
G(n, k, a) & =\frac{1}{(k-1) !} \sum_{j=1}^{k}\left[\begin{array}{l}
k \\
j
\end{array}\right](-1)^{k-j} a^{j-1}\left(-0^{j-1}\right) \\
& =\frac{1}{(k-1) !}\left[\begin{array}{c}
k \\
1
\end{array}\right](-1)^{k-1} a^{0}\left(-0^{0}\right) \\
& =(-1)^{k} .
\end{aligned}
$$

Therefore $F(n, k)=\sum_{a=1}^{n}(-1)^{a}\left(\begin{array}{l}n \\ a\end{array}\right)(-1)^{k}=(-1)^{k}\left((1-1)^{n}-1\right)=(-1)^{k-1}$. This allows us to write Equation 14 as $(-1)^{k-1} / k$ which, in turn, means that Equation 13 is

$$
\left(\mathfrak{R}^{(W)}\right)_{D, D^{\prime}}^{2}=\sum_{k=1}^{n} \sum_{\substack{\gamma \in \text { Colours }_{k}(W) \\ \gamma(D)=D^{\prime}}} \frac{(-1)^{k-1}}{k}=\mathfrak{R}_{D, D^{\prime}}^{(W)}
$$

\section{Indecomposable permutations and a web world on two pegs having multiple edges}

In this section we consider the web world $W_{n}$ whose web graph is $G\left(W_{n}\right)=K_{2}$ and the single edge is labelled with $n$. This web world consists of diagrams having two pegs and $n$ edges connecting the $n$ vertices on the first peg to $n$ vertices on the second peg. From a physics point of view, such webs have been studied for a long time [13, 7, 19]. However, they have yet to be analysed using the language of web-colouring and mixing matrices and which we will now attend to.

Every diagram $D \in W_{n}$ is uniquely described as a set of 4-tuples

$$
D=\left\{\left(1,2, i, \pi_{i}\right): \pi \in \mathcal{S}_{n}\right\}
$$

and consequently there are $n$ ! diagrams in $W_{n}$. We can abbreviate this by writing $D=D_{\pi}$. Given a permutation $\pi=\pi_{1} \ldots \pi_{n}$ and permutations $\alpha \in \mathcal{S}_{\ell}, \beta \in \mathcal{S}_{n-\ell}$, we say that $\pi$ is the sum of the permutations $\alpha$ and $\beta$ if $\pi_{i}=\alpha_{i}$ for all $i \leqslant \ell$ and $\pi_{i}=\ell+\beta_{i-\ell}$ for all $i>\ell$. We write this as $\pi=\alpha+\beta$.

If a permutation $\pi$ can be written as such a sum of smaller permutations, then we say that $\pi$ is decomposable. Otherwise we say that $\pi$ is indecomposable. Let $\operatorname{lnd}_{n}$ be the set of indecomposable permutations of length $n$ and let Ind $=\cup_{n \geqslant 1} \operatorname{lnd}_{n}$. Every permutation $\pi \in \mathcal{S}_{n}$ admits a unique representation as a sum of indecomposable permutations. If $\pi=\sigma_{1}+\cdots+\sigma_{k}$ where every $\sigma_{i} \in$ Ind, then $D_{\pi}=D_{\sigma_{1}} \oplus \cdots \oplus D_{\sigma_{k}}$ and we will write indparts $(\pi)$ for the number $k$ of indecomposable permutations in the sum. 
Problem 25. [Word reconstruction generating function]

Given a finite word $w=w_{1} \ldots w_{n}$ on a finite alphabet, let $f_{w}(k)$ be the number of ways to read the word from left to right $k$ times (each time always picking some unchosen letter) so that we arrive back at the original word $w$. Let $F_{w}(x)=\sum_{k} f_{w}(k) x^{k}$.

Example 26. Let $w=c b a b a c=w_{1} w_{2} w_{3} w_{4} w_{5} w_{6}$ be a word. The number of ways to read $w$ from left to right in one passing is 1 since we must sequentially read all letters $w_{1} w_{2} w_{3} w_{4} w_{5} w_{6}$, so $f_{w}(1)=1$. There are several ways to read $w$ from $w$ in two passings: read positions 1 through to 5 on the first passing, and read position 6 on the second passing. This is summarized by the reading vector $(1,1,1,1,1,2)$ where $j$ at position $i$ means $w_{i}$ is read on the $j$ th passing. If we abbreviate this to 111112 , then the other reading vectors for $k=2$ are 111122, 111222, 112222, 122222, and 122112 so $f_{w}(2)=6$. For this case, $F_{w}(x)=x+6 x^{2}+17 x^{3}+26 x^{4}+22 x^{5}+8 x^{6}$.

Example 27. If all letters of a length $n$ word $w$ are distinct, then $F_{w}(x)=x(1+$ $x)^{n-1}$. Similarly if $w$ is a word consisting of $n$ copies of the same letter, then $F_{w}(x)=$ $\sum_{k=1}^{n} k !\left\{\begin{array}{l}n \\ k\end{array}\right\} x^{k}=\mathcal{F}_{n}(x)$.

Theorem 28. Let $D_{\pi} \in W_{n}$ with $\pi=w_{1} \oplus \cdots \oplus w_{n}$ where every $w_{i} \in$ Ind. Let $w$ be the word $\left(w_{1}, \ldots, w_{\text {indparts }(\pi)}\right)$ on the alphabet $\left\{w_{1}, \ldots, w_{\text {indparts }(\pi)}\right\}$. Then $\mathfrak{M}_{D_{\pi}, D_{\pi}}^{(W)}(x)=F_{w}(x)$.

Proof. Let $D_{\pi} \in W_{n}$ with $\pi=\sigma_{1} \oplus \cdots \oplus \sigma_{m}$. In order to colour the edges of $D_{\pi}$ so that the same diagram is reconstructed, the colours of the edges that are in the same indecomposable part must remain the same. (Otherwise we would be constructing a web diagram which has more indecomposable parts than the original one, which would be a contradiction.) Given a set $A=\left\{a_{1}, \ldots, a_{i}\right\}$, define $\sigma_{A}=\sigma_{a_{1}} \oplus \cdots \oplus \sigma_{a_{i}}$ where $a_{1}<\cdots<a_{i}$. Let

$$
\text { Colourings }(m, k)=\left\{\left(c_{1}, \ldots, c_{m}\right) \in[1, k]^{m}:\left\{c_{1}, \ldots, c_{m}\right\}=[1, k]\right\} .
$$

Given $c \in$ Colourings $(m, k)$, let $\operatorname{Col}_{c}(i)=\left\{j \in[1, m]: c_{j}=i\right\}$.

The set of colourings $c^{\prime}$ of edges of $D_{\pi}$ which results in a reconstruction of the diagram is in 1-1 correspondence with the set of colourings $c \in \operatorname{Colourings}(m, \cdot)$ such that

$$
\sigma_{1} \oplus \cdots \oplus \sigma_{m}=\sigma_{\mathrm{Col}_{c}(1)} \oplus \cdots \oplus \sigma_{\mathrm{Col}_{c}(k)} .
$$

This is the word reconstruction problem (Problem 25) for the word $w=\left(\sigma_{1}, \ldots, \sigma_{m}\right)$ which is on the alphabet $\left\{\sigma_{1}, \ldots, \sigma_{m}\right\}$.

There is no simple formula for the diagonal entries of the web-colouring matrix for this web world due to there being no 'closed form' answer to Problem 25. However, we have noticed some potential structure from looking at the trace of the web-colouring matrices for small values of $n$, outlined in Figure 7. The numbers from Figure 7 support the following conjecture for all $n \in[1,8]$.

Conjecture 29. Let $a(n)=\left[x^{n-1}\right]$ trace $\left(\mathfrak{M}^{(W(n))}(x)\right)$. Then $a(n)=(n-2) !\left(n^{2}-3 n+4\right) / 2$ for all $n>1$. This sequence of numbers enumerates a class of directed column convex polyominoes in the plane. (See [17, A121635] and [1].) 


\begin{tabular}{|c|c|}
\hline$n$ & $\operatorname{trace}\left(\mathfrak{M}^{(W(n))}(x)\right)$ \\
\hline \hline 1 & $x$ \\
2 & $2 x+2 x^{2}$ \\
3 & $6 x+8 x^{2}+6 x^{3}$ \\
4 & $24 x+30 x^{2}+42 x^{3}+24 x^{4}$ \\
5 & $120 x+116 x^{2}+216 x^{3}+264 x^{4}+120 x^{5}$ \\
6 & $720 x+532 x^{2}+1002 x^{3}+1920 x^{4}+1920 x^{5}+720 x^{6}$ \\
7 & $5040 x+2848 x^{2}+4626 x^{3}+11688 x^{4}+19200 x^{5}+15840 x^{6}+5040 x^{7}$ \\
\hline
\end{tabular}

Figure 7: The sequence of numbers that correspond to [17, A121635] (as mentioned in Conjecture 29) are the coefficients of the second highest exponent of $x$ in each row: 2, 8, 42, 264, 1920 and 15840 .

\section{References}

[1] E. Barcucci, A. Del Lungo, and R. Pinzani. "Deco" polyominoes, permutations and random generation. Theor. Comput. Sci. 159(1):29-42, 1996.

[2] P. J. Cameron, D. A. Gewurz, and F. Merola. Product action. Discrete Math. 308:386-394, 2008.

[3] P. J. Cameron, T. Prellberg, and D. Stark. Asymptotic enumeration of incidence matrices. J. Phys. Conf. Ser. 42:59-70, 2006.

[4] M. Dukes, E. Gardi, H. McAslan, D. J. Scott, and C. D. White. Webs and posets. J. High Energy Phys. 2014(1), Article 024, 2014.

[5] M. Dukes, E. Gardi, E. Steingrímsson, and C. D. White. Web worlds, web-colouring matrices, and web-mixing matrices. J. Combin. Theory Ser. A 120(5):1012-1037, 2013.

[6] G. Falcioni, E. Gardi, M. Harley, L. Magnea, and C. D. White. Multiple Gluon Exchange Webs. J. High Energy Phys. 2014(10), Article 010, 2014.

[7] J. Frenkel and J. C. Taylor. Nonabelian Eikonal Exponentiation. Nucl. Phys. B 246(2): 231-245, 1984.

[8] E. Gardi. From Webs to Polylogarithms. J. High Energy Phys. 2014(4), Article 044, 2014.

[9] E. Gardi, E. Laenen, G. Stavenga, and C. D. White. Webs in multiparton scattering using the replica trick. J. High Energy Phys. 2010(11), Article 155, 2010.

[10] E. Gardi, J. M. Smillie, and C. D. White. On the renormalization of multiparton webs. J. High Energy Phys. 2011(9), Article 114, 2011.

[11] E. Gardi, J. M. Smillie, and C. D. White. The Non-Abelian Exponentiation theorem for multiple Wilson lines. J. High Energy Phys. 2013(06), Article 088, 2013.

[12] E. Gardi and C. D. White. General properties of multiparton webs: proofs from combinatorics. J. High Energy Phys. 2011(3), Article 079, 2011. 
[13] J. G. M. Gatheral. Exponentiation of Eikonal Cross-sections in Nonabelian Gauge Theories. Phys. Lett. B 133(1\&2):90-94, 1983.

[14] R. L. Graham, D. E. Knuth, and O. Patashnik. Concrete Mathematics: A Foundation for Computer Science. 2nd edition. Addison-Wesley Longman Publishing Co., 1994.

[15] M. Maia and M. Mendez. On the arithmetic product of combinatorial species. Discrete Math. 308(23):5407-5427, 2008.

[16] A. Mitov, G. Sterman, and I. Sung. Diagrammatic Exponentiation for Products of Wilson Lines. Phys. Rev. D 82, Article 096010, 2010.

[17] The On-Line Encyclopedia of Integer Sequences, published electronically at http://oeis.org, 2010.

[18] R. P. Stanley. Two Poset Polytopes. Discrete Comput. Geom. 1(1):9-23, 1986.

[19] G. F. Sterman. Infrared Divergences in Perturbative QCD. AIP Conf. Proc. 74:22-40, 1981.

[20] A. A. Vladimirov. Exponentiation for products of Wilson lines within the generating function approach. J. High Energy Phys. 2015(6), Article 120, 2015.

[21] Eric W. Weisstein. Stirling Number of the Second Kind. From MathWorld - A Wolfram Web Resource. http://mathworld.wolfram.com/StirlingNumberoftheSecondKind.html

[22] C. D. White. An Introduction to Webs. J. Phys. G: Nucl. Part. Phys. 43(3), Article $033002,2016$. 